\title{
Competing mechanisms for singlet-triplet transition in artificial molecules
}

\author{
Devis Bellucci,* Massimo Rontani, Filippo Troiani, Guido Goldoni, and Elisa Molinari \\ INFM-National Research Center on nano-Structures and bio-Systems at Surfaces $\left(S^{3}\right)$ and Dipartimento di Fisica, \\ Università degli Studi di Modena e Reggio Emilia, Via Campi 213/A, 41100 Modena, Italy
}

(Received 27 February 2004; published 20 May 2004)

\begin{abstract}
We study the magnetic field induced singlet/triplet transition for two electrons in vertically-coupled quantum dots by exact diagonalization of the Coulomb interaction. We identify the different mechanisms occurring in the transition, involving either in-plane correlations or localization in opposite dots, depending on the field direction. Therefore, both spin and orbital degrees of freedom can be manipulated by field strength and direction. The phase diagram of realistic devices is determined.
\end{abstract}

DOI: 10.1103/PhysRevB.69.201308

PACS number(s): 73.21.La, 73.23.Hk

Atomic-like phenomenology, ensuing from the discrete density of states, has been predicted and demonstrated in semiconductor quantum dots (QDs), such as shell structure, ${ }^{1,2}$ fine structure due to exchange interaction (Hund's rule), ${ }^{3}$ and Kondo physics; ${ }^{4}$ hence QDs are often termed artificial atoms. Carriers can be injected one by one into the system in single-electron transport ${ }^{2}$ or capacitance ${ }^{1}$ experiments, based on the Coulomb blockade ${ }^{5}$ phenomenon, and the energy required to add one electron can be measured if the electrostatic screening is poor and the thermal smearing is low.

Coupled QDs extend to the molecular realm the similarity between natural and artificial atoms; ${ }^{6,7}$ here, inter-dot tunneling introduces an energy scale which may be comparable to other energy scales of the system, namely, single-particle confinement energy, carrier-carrier interaction, and magnetic energy. In contrast to natural molecules, where inter-nuclear coupling is fixed by the balance between nuclear repulsion and electrostatic attraction mediated by valence electrons, in such artificial molecules (AM) all energy scales, including inter-dot coupling, as well as the charging state of the system can be controlled to a very high degree by device engineering and/or external fields. ${ }^{8}$

A typical AM consists of a disc-like region obtained from coupled two-dimensional quantum systems, such as two quantum wells (vertically coupled QDs). As in single QDs, electronic states can be easily manipulated by a magnetic field $B_{\perp}$, perpendicular to the plane of the QDs, which drives the system from a low-correlation (low-field) regime to a highly correlated (high-field) regime by changing the single-particle splittings. ${ }^{9}$ The study of electronic states of few electrons in $\mathrm{AMs}^{10,11}$ has become a topic of increasing interest, partially due to possible implications for the implementation of scalable solid-state quantum gates, with the quantum bit of information coded either in the electron charge $^{12}$ or $\operatorname{spin}^{13}$ degree of freedom (DOF).

It should be noted that in AMs carriers are not only electrostatically coupled, but also have their spin interlaced when tunneling is allowed, ${ }^{14}$ since electrons with opposite spin may tunnel into the same dot if the intra-dot Coulomb interaction is not too large; the same process is obviously prohibited for electrons with parallel spins. This two-electron dynamics may be described by an effective Heisenberg Hamiltonian $H=J(\mathbf{B}) \mathbf{s}_{1} \cdot \mathbf{s}_{2}$ between spins $\mathbf{s}_{1}$ and $\mathbf{s}_{2}$, ${ }^{14}$ with singlet and triplet configurations separated by a fielddependent exchange-energy gap $J(\mathbf{B}) \equiv E_{t}-E_{s}$, which is positive at zero field. ${ }^{15}$ One convenient way to control interdot tunneling, and, hence, effective spin-spin interaction $J$, is by applying a magnetic field with a finite component in the plane of the QDs, i.e., perpendicular to the tunneling direction $B_{\|} \cdot{ }^{14,16}$ Controlling tunneling by $B_{\|}$has the advantage that other energy scales and, in particular, the Coulomb interaction are practically unaffected. However, few studies are devoted to this field configuration, which lacks the cylindrical symmetry which can be exploited in the vertical field arrangement. On the other hand, controlling $J(\mathbf{B})$ in AM is crucial for the proposed implementation of scalable quantum gates. $^{14}$

In this paper we study the exchange energy for two electrons confined in AMs in a magnetic field of arbitrary direction. This is performed by a fully numerical, real-space approach which allows one to account for the complexity of realistic samples; the carrier-carrier Coulomb Hamiltonian is diagonalized exactly within a large single-particle basis. We show that the field drives the system from an uncorrelated regime, where the singlet state is stable, to a strongly correlated one, where triplet ordering is favored; however, the transition occurs by different mechanisms, whether the field is in the vertical or in the in-plane direction.

We consider two electrons in a general QD structure. Carriers are described by the effective-mass Hamiltonian

$$
\begin{aligned}
H= & \sum_{i=1}^{N}\left[-\frac{\hbar^{2}}{2 m^{*}}\left(\nabla_{i}+\frac{e}{c} \mathbf{A}\left(\mathbf{r}_{i}\right)\right)^{2}+V\left(\mathbf{r}_{i}\right)\right] \\
& +\frac{1}{2} \sum_{\substack{i, j=1 \\
i \neq j}}^{N} \frac{e^{2}}{\epsilon^{*}\left|\mathbf{r}_{i}-\mathbf{r}_{j}\right|}+g^{*} \mu_{B} \mathbf{B} \cdot \mathbf{S}
\end{aligned}
$$

with $N=2$. Here $m^{*}, \epsilon^{*}$, and $g^{*}$ are the effective mass, dielectric constant, and $g$-factor, respectively. ${ }^{17}$ Equation (1) neglects non-parabolicity effects, but otherwise includes the full three-dimensional (3D) nature of the quantum states in realistic samples, such as layer width and finite band offsets, by the effective potential $V(\mathbf{r})$. Our numerical approach consists in mapping the single-particle terms in a real-space grid, leading to a large sparse matrix which is diagonalized by the Lanczos method. Single-particle spin-orbitals are then used 


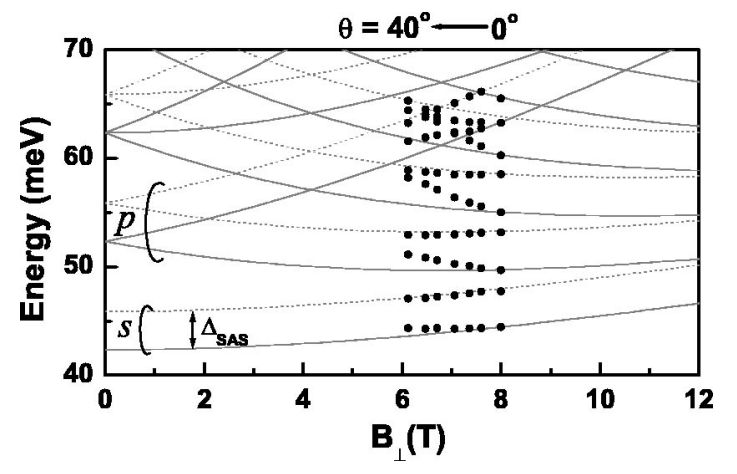

FIG. 1. Single-particle energy levels for a GaAs AM in a magnetic field. Solid and dotted lines represent the FD states $\varepsilon_{n m}$ induced by a strictly vertical field for S and AS levels, respectively. $s$ and $p$ shells (see Ref. 21) are indicated. Dots represent calculated energy levels for a total field of $8 \mathrm{~T}$, rotated from $0^{\circ}$ to $40^{\circ}$ with respect to the AM vertical axis. Sample parameters are as follows: $L_{W}=10 \mathrm{~nm}, L_{B}=3 \mathrm{~nm}, V_{0}=300 \mathrm{meV}$, and $\hbar \omega_{0}=10 \mathrm{meV}$.

to build a basis of Slater determinants for the $N$-particle problem, which is then used to represent the two-body term, in the familiar configuration interaction approach. ${ }^{7}$ Coulomb matrix elements are calculated numerically. The ensuing matrix, which can be very large, is again sparse and can be diagonalized via the Lanczos method as well. ${ }^{18}$

In the following the potential $V(\mathbf{r})$ describes two identical vertically coupled disk-like QDs. As usual for this type of samples which have very different confinement energies in the growth and in-plane directions, we separate the potential as $V(x, y)+V(z)$, where $V(z)$ represent two symmetric quantum wells of width $L_{W}$ separated by a barrier $L_{B}$ and conduction band mismatch $V_{0}$. We perform the common choice of a parabolic in-plane confinement $(1 / 2) m^{*} \omega_{0}^{2}\left(x^{2}\right.$ $\left.+y^{2}\right)$, as this has proved to be quantitatively accurate. ${ }^{11}$ Note, however, that our numerical approach does not assume any symmetry; in particular, the vector potential $\mathbf{A}(\mathbf{r})$ is not limited to describe $z$-directed field.

In a QD with parabolic in-plane confinement and strictly perpendicular magnetic field, single-particle states are given by the Fock-Darwin (FD) states (see, e.g., Ref. 19), with energies $\varepsilon_{n m}=\hbar \Omega(2 n+|m|+1)-\left(\hbar \omega_{c} / 2\right) m, n$ and $m$ being the principal and azimuthal quantum numbers, respectively. The oscillator frequency is $\Omega=\sqrt{\omega_{0}^{2}+\omega_{c}^{2} / 4}$, with the cyclotron frequency $\omega_{c}=e B / m^{*} c$. In symmetric AMs we have two such ladders of energy levels, associated with the symmetric (S) and anti-symmetric (AS) states arising from the double-well potential in the growth direction, rigidly separated by a splitting $\Delta_{\text {SAS }}$ (see Fig. 1 ).

We next consider the effect of a magnetic field with a finite in-plane component $B_{\|}$. As shown in Fig. 1, when the angle $\theta$ between a fixed $|\mathbf{B}|$ and the $z$ axis is increased, the energy levels no longer correspond to the FD states at the corresponding $B_{\perp}$. Indeed, the splitting between $\mathrm{S}$ and $\mathrm{AS}$ levels decreases with increasing $\theta,{ }^{20}$ which shows that an in-plane component of the field suppresses the tunneling; note that this effect is larger for higher levels. It is important to stress that the in-plane field can meaningfully affect the motion along the growth direction if $\omega_{c}^{\|}=e B_{\|} / m^{*} c$
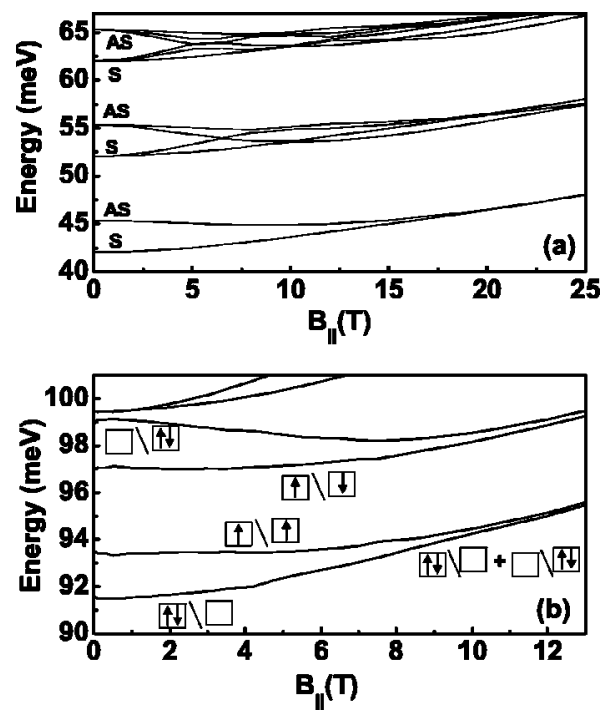

FIG. 2. Energy levels vs in-plane field at $B_{\perp}=0$ for the same AM of Fig. 1. (a) Single-particle levels, with indication of the S/AS character at low field. (b) Two-electron levels. Insets: main components of the wave functions in terms of S (left boxes) and AS (right boxes) single-particle states.

$\sim \Delta_{\text {SAS }}$. Similar effects are much harder to achieve in single QDs, due to the large single-particle gaps induced by the single quantum well confinement.

As discussed in more detail in the following, the reduction of the energy gap between the $s$ and the $p$ shells ${ }^{21}$ (see Fig. 1) strongly reduces the single-particle energy of the triplet state with respect to that of the singlet: the perpendicular field thus promotes the singlet-triplet crossing. This transition results in an enhancement of the in-plane correlation of the twoelectron ground state and in the spin-polarization of the system, ${ }^{19,22}$ arising from the exchange (orbital) interaction. ${ }^{23}$ Note that this mechanism only involves the in-plane DOFs, and is therefore present in both single and coupled QDs; in order to observe some marked differences in the behavior of the two systems, one needs to excite the motion along the growth direction $z$.

Figure 2(a) shows the single-particle levels as a function of the in-plane field $B_{\|}$. The energy levels come in shells with $\mathrm{S}$ and AS character, but the degeneracies which are present at $B_{\|}=0$ are removed by a finite field, as the axial symmetry of the system is lost. Therefore, the single-particle wave functions do not have a well defined angular momentum, and are now $\mathrm{S}$ or AS only with respect to a $180^{\circ}$ rotation about the axis parallel to $\mathbf{B}$. Besides, as the field is increased, the $\mathrm{S}$ and AS levels approach each other, since the tunneling is progressively suppressed. ${ }^{16}$

In Fig. 2(b) we show the lowest two-particle levels, and schematically indicate the main components of the corresponding wave functions in terms of $\mathrm{S}$ and AS single-particle states. At low $B_{\|}$the ground- and the first excited-state have a singlet and a triplet character, respectively. As $B_{\|}$is increased, the energy gap $J$ is suppressed: indeed, singlet and triplet states have the same orbital energy, while the Zeeman term favors the latter (in the field range of Fig. 2 the Zeeman contribution can hardly be distinguished). As shown in the 

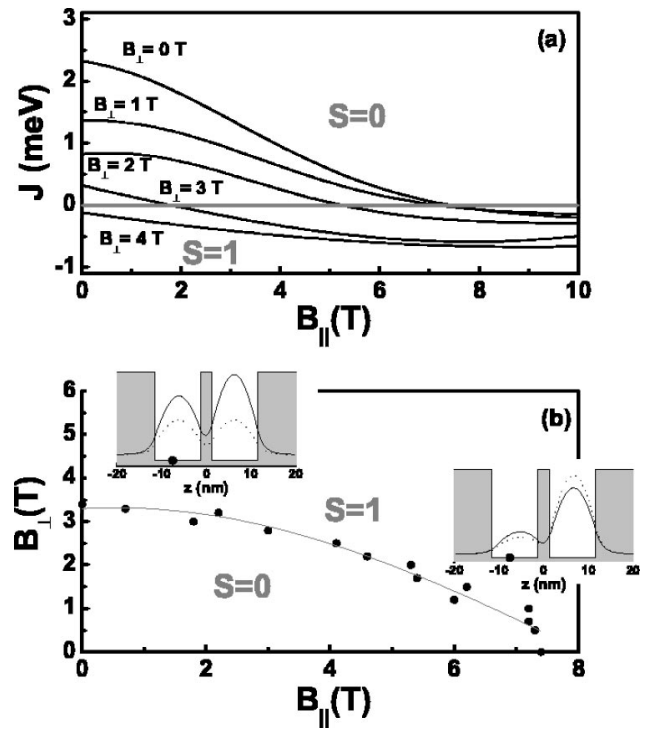

FIG. 3. (a) Exchange energy $J$ vs in-plane field at selected vertical fields, for a GaAs AM. Sample parameters are as in Fig. 1, but for a weaker lateral confinement $\hbar \omega_{0}=4 \mathrm{meV}$. For clarity we show the best fitting curves from a large number of calculated points. Numerical inaccuracies may result in $\pm 0.3 \mathrm{meV}$ shift from the curves only for the highest $B_{\perp}$. (b) Calculated singlet/triplet phase diagram. The line is a guide to the eye through the calculated points (Ref. 26). Insets show the singlet (solid line) and triplet (dashed line) conditional probability near the transitions, defined as $\left|\psi\left(\boldsymbol{\rho}_{0}, z_{0}, \boldsymbol{\rho} ; z\right)\right|^{2} ; \psi$ is the two-electron wave function, with $\boldsymbol{\rho}$ the in-plane coordinate with respect to the vertical axis of the cylindrical QD. The reference electron (black dot) is fixed at $z_{0}=$ $-7.5 \mathrm{~nm}$, at an in-plane position $\left|\boldsymbol{\rho}_{0}\right|=4.4 \mathrm{~nm}$; the conditional probability is then plotted along an axis parallel to $z$ and crossing the QD plane at a position diametrically opposed to the reference electron. Left inset: $B_{\perp}=4 \mathrm{~T}, B_{\|}=0 \mathrm{~T}$. Right inset: $B_{\perp}=0 \mathrm{~T}, B_{\|}$ $=9 \mathrm{~T}$.

insets, the transition occurs with the maximal, Coulombinduced mixing of the S and AS states, which is favored by the vanishing of $\Delta_{\mathrm{SAS}}$ at large fields. In other words, increasing $B_{\|}$the singlet state evolves from a nearly pure $\mathrm{S}$ state to a fully entangled state in the S/AS basis. Note also that, contrary to the one occurring at large $B_{\perp}$, here the transition is associated to the correlation along the growth direction, i.e., with the two electrons sitting on opposite QDs, as we will show below.

The ability to control both the exchange energy $J$ and the effective Hilbert-space structure is indeed pivotal to the QDbased implementations of quantum-information processing ${ }^{14}$ besides, $J$ is of direct experimental interest, for it can be probed by single-electron excitation spectroscopy. ${ }^{24}$ In Fig. 3 (a) we show the calculated exchange energy as a function of the in-plane field at different values of $B_{\perp}$ and for a weaker parabolic confinement $\left(\Delta_{S A S} \leqslant \hbar \omega_{0}\right)$. The positive/negative $J$ region is the stability region for singlet/triplet states. Figure 3(a) shows that an increase in $B_{\perp}$ monotonically $(i)$ reduces the singlet stability range with respect to $B_{\|}$, and (ii) enhances the ferromagnetic $(J<0)$ behavior in the considered range of $B_{\|}$values. These features are summarized in the phase diagram shown in Fig. 3(b).
A closer inspection into our results shows that different mechanisms are involved in the singlet/triplet transition, depending on the field direction. At zero field the singlet state mainly corresponds to both electrons occupying the $(s, S)$ orbital, while a minor contribution from the $(s, \mathrm{AS})$ orbital gives rise to the spatial correlation in the $z$ direction. On the contrary, all the dominant configurations in the triplet state involve S states (see the conditional probability in Fig. 3(b)). $B_{\perp}$ leaves unaffected the $z \mathrm{DOF}$, while it energetically lowers the $p(m=1)$ state with respect to the $s$ one. The positive single-particle contribution to $J$ is therefore reduced, until it is compensated by the negative contribution arising from the Coulomb energy. To summarize, the singlet/triplet crossing induced by $B_{\perp}$ is mainly connected with the in-plane dynamics, while it leaves unaffected the motion in the growth direction and the double-occupancy probability of each dot.

The main effect of $B_{\|}$(right inset), instead, is that of suppressing the energy splitting resulting from the interdot tunneling. This clearly favors the occupation of the AS states, and therefore vertical correlations for both the singlet and the triplet states set in; in both cases, the two electrons tend to localize in opposite dots, and the importance of the spins' relative orientation vanishes with the double occupancy probability. Indeed the exponential vanishing of $J$ represents the clear fingerprint of the regime where the doubleoccupancy probability is suppressed. It should be noted that this is not a single-particle effect, since it does not imply, nor require, the complete suppression of the tunneling.

The results reported in Fig. 3 show that these two different mechanisms interfere with each other in a non-trivial manner. The presence of the perpendicular component $B_{\perp}$ favors the single-triplet crossing and the ferromagnetic phase, while it opposes the suppression of the double occupancy and the resulting singlet-triplet degeneracy (apart from the Zeeman term). Such interplay arises from the 3D nature of quantum states in the AM: in fact, in the considered range of physical parameters $\left(\hbar \omega_{0} \sim \Delta_{\text {SAS }}\right)$, the magnetic field can strongly affect both the in-plane (intra-dot) and the vertical (inter-dot) DOF.

An adiabatic manipulation of $J$ by means of magnetic (and electric) fields has been proposed in order to implement the two-qubit gates in electron-spin based quantum computers. ${ }^{14}$ The rest condition within such scheme would correspond to the suppression of $J$ and of the overlap between electrons localized in adjacent QDs, where both conditions should be induced by a static magnetic field. In this perspective, our findings suggest that $(i)$ the $B_{\|}$(rather than $B_{\perp}$ ) component of the field and the exponential suppression (rather than the crossing point from $J>0$ to $J<0$ ) are required; $(i i)$ the presence of a field component perpendicular to the static one (as required, e.g., for the single-spin rotations) should be simultaneously taken into account in order to determine the suited range of physical parameters.

To summarize, we have theoretically investigated the dependence of the singlet and triplet states of two electrons in AMs on external magnetic fields of arbitrary direction. Our computational approach allows one to fully account for the different physical mechanisms underlying the singlet/triplet transitions which are due to the parallel and perpendicular 
components of the field, as well as for the non-trivial interplay between the vertical and the in-plane correlation effects that they induce. The perpendicular component of the field does indeed facilitate the transition from the antiferromagnetic to the ferromagnetic phase which is induced by the parallel component, but at the same time it opposes the carrier localization and correlation properties that the latter tends to induce in the AM.
We are grateful to S. Amaha, D. G. Austing, A. Bertoni, U. Hohenester, V. Pellegrini, S. Tarucha, and C. Tejedor for useful discussions. This work was supported in part by MIUR FIRB-RBAU01ZEML Quantum phases of ultra-low electron density semiconductor heterostructures, and by INFM-IT Calcolo Parallelo. With the contribution of the Italian Minister for Foreign Affairs, Direzione Generale per la Promozione e la Cooperazione Culturale.
*Electronic address: dbellucci@unimore.it; www.nanoscience.unimore.it

${ }^{1}$ R. Ashoori, Nature (London) 379, 413 (1996).

${ }^{2}$ S. Tarucha, D.G. Austing, T. Honda, R.J. van der Hage, and L.P. Kouwenhoven, Phys. Rev. Lett. 77, 3613 (1996).

${ }^{3}$ S. Tarucha, D.G. Austing, Y. Tokura, W.G. van der Wiel, and L.P. Kouwenhoven, Phys. Rev. Lett. 84, 2485 (2000).

${ }^{4}$ D.G. Goldhaber-Gordon, H. Shtrikman, D. Mahalu, D. AbuschMagder, U. Meirav, and M.A. Kastner, Nature (London) 391, 156 (1998); S.M. Cronenwett, T.H. Oosterkamp, and L.P. Kouwenhoven, Science 281, 540 (1998).

${ }^{5}$ Single charge tunneling: Coulomb blockade phenomena in nanostructures, edited by H. Grabert and M.H. Devoret, NATO ASI series B, Vol. 294 (Plenum, New York, 1992).

${ }^{6}$ L.P. Kouwenhoven, Science 268, 1440 (1995).

${ }^{7}$ M. Rontani, F. Troiani, U. Hohenester, and E. Molinari, Solid State Commun. 119, 309 (2001).

${ }^{8}$ W.G. van del Wiel, S. De Franceschi, J.M. Elzerman, T. Fujisawa, S. Tarucha, and L.P. Kouwenhoven, Rev. Mod. Phys. 75, 1 (2003); M. Rontani, S. Amaha, K. Muraki, F. Manghi, E. Molinari, S. Tarucha, and D.G. Austing, Phys. Rev. B 69, 085327 (2004)

${ }^{9}$ M. Rontani, G. Goldoni, F. Manghi, and E. Molinari, Europhys. Lett. 58, 555 (2002).

${ }^{10}$ J.J. Palacios and P. Hawrylak, Phys. Rev. B 51, 1769 (1995); J.H. Oh, K.J. Chang, G. Ihm, and S.J. Lee, ibid. 53, R13264 (1996); M. Rontani, F. Rossi, F. Manghi, and E. Molinari, Solid State Commun. 112, 151 (1999); Y. Tokura, D.G. Austing, and S. Tarucha, J. Phys.: Condens. Matter 11, 6023 (1999); B. Partoens and F.M. Peeters, Phys. Rev. Lett. 84, 4433 (2000); L. MartinMoreno, L. Brey, and C. Tejedor, Phys. Rev. B 62, R10633 (2000); M. Pi, A. Emperador, M. Barranco, F. Garcias, K. Muraki, S. Tarucha, and D.G. Austing, Phys. Rev. Lett. 87, 066801 (2001); M. Rontani, G. Goldoni, and E. Molinari, in New directions in mesoscopic physics (towards nanoscience), edited by R. Fazio, V. F. Gantmakher, and Y. Imry, NATO ASI series B, Vol. 125 (Kluwer, Dordrecht, 2003), p. 361; cond-mat/0212626.

${ }^{11}$ S.M. Reimann and M. Manninen, Rev. Mod. Phys. 74, 1283 (2002).

${ }^{12}$ A. Barenco, D. Deutsch, A. Ekert, and R. Jozsa, Phys. Rev. Lett. 74, 4083 (1995).
${ }^{13}$ D. Loss and D.P. DiVincenzo, Phys. Rev. A 57, 120 (1998).

${ }^{14}$ G. Burkard, G. Seelig, and D. Loss, Phys. Rev. B 62, 2581 (2000).

${ }^{15}$ The exchange energy $J\left(B_{\perp}, B_{\|}\right)$results from both single-particle and Coulomb contributions. The term exchange interaction, as used in the paper, indicates only the Coulomb term depending on the symmetry of the two-electron spatial wave function.

${ }^{16}$ Y. Tokura, S. Sasaki, D.G. Austing, and S. Tarucha, Physica E (Amsterdam) 6, 676 (2000).

${ }^{17}$ We use standard values of GaAs bulk parameters: $m^{*} / m_{0}$ $=0.067, \epsilon^{*}=12.9, g^{*}=-0.44$.

${ }^{18}$ Typical calculations are performed on a $(100 \times 100 \times 40) \mathrm{nm}$ grid with $(64 \times 64 \times 128)$ points along directions $(x, y, z)$, respectively; the Coulomb interaction is represented on a singleparticle basis set consisting of 12 states.

${ }^{19}$ U. Merkt, J. Huser, and M. Wagner, Phys. Rev. B 43, 7320 (1991).

${ }^{20}$ Here the S/AS labeling is used for brevity. Obviously, for a general field direction with respect to the tunneling direction, wave functions do not have a well defined S/AS symmetry. See Bellucci et al. (Ref. 25).

${ }^{21}$ We classify the AM states according to their vertical (S/AS) and in-plane (FD) character. The $s$ and $p$ shells correspond to the $(n, m)=(0,0)$ and $(0, \pm 1)$ FD states, respectively. In the literature, AM states are sometimes classified according to the molecular notation: the $s$ and $p$ states with $\mathrm{S}$ (AS) character correspond to $\sigma_{g}, \pi_{u}\left(\sigma_{u}, \pi_{g}\right)$ molecular orbitals, respectively. See Rontani et al. (Ref. 7).

${ }^{22}$ D. Pfannkuche, V. Gudmundsson, and P.A. Maksym, Phys. Rev. B 47, 2244 (1993).

${ }^{23}$ Eventually, as the field is further increased, electron localize in a confined counterpart of a Wigner crystal. See Rontani et al. (Ref. 9).

${ }^{24}$ L.P. Kouwenhoven, T.H. Oosterkamp, M.W.S. Danoesastro, M. Eto, D.G. Austing, T. Honda, and S. Tarucha, Science 278, 1788 (1997).

${ }^{25}$ D. Bellucci, M. Rontani, G. Goldoni, F. Troiani, and E. Molinari, Physica E (Amsterdam) 22, 482 (2004).

${ }^{26}$ Scattering of calculated points is related to the finiteness of the space grids. These inaccuracies are more significant for nonsymmetric field directions. 